\title{
CAPITAL HUMANO, DESIGUALDAD Y CRECIMIENTO ECONÓMICO EN AMÉRICA LATINA*
}

\author{
Luis Brito Gaona \\ Emma Iglesias Vásquez
}

* DOI: https://doi.org/10.18601/01245996.v23n45.13. Recepción: 21-012019, modificación final: 30-04-2021, aceptación: 02-06-2021. Sugerencia de citación: Brito, L. F. e Iglesias, E. M. (2021). Capital humano, desigualdad y crecimiento económico en América Latina. Revista de Economía Institucional, 23(45), 265-283.

a Doctor en Economía. Profesor de la Facultad de Ciencias Empresariales, Universidad Técnica de Machala, E1 Oro, Ecuador, [1brito@utmachala.edu.ec].

b Doctora en Economía. Catedrática del Departamento de Economía, Universidad de A Coruña, A Coruña, España, [emma.iglesias@udc.es]. 


\section{Capital humano, desigualdad y crecimiento económico en América Latina}

Resumen Macarena Suanes y Oriol Roca estudiaron los principales determinantes de la desigualdad y el crecimiento economico en America-Latina entre 1980 y 2009 , y encontraron que uno de los principales fue la inversion extranjera directa. Este articulo extiende ese estudio hasta 2015, y muestra que dicha inversion ya no es significativa. En cambio, el aumento del capital humano reduce en forma significativa la desigualdad y mejora el crecimiento economico. Se recomienda, entonces, que las politicas economicas se orienten a elevar el nivel educativo.

Palabras clave: crecimiento económico, capital humano, desigualdad, América Latina; JEL: I24, I25, I28

\section{Human capital, inequality and economic growth in Latin-America}

Abstract Macarena Suanes and Oriol Roca studied the main determinants of inequality and economic growth in Latin America between 1980 and 2009 and found that one of the main determinants was foreign direct investment. This paper extends that study to 2015 and shows that such investment is no longer significant. In contrast, increasing human capital significantly reduces inequality and improves economic growth. It is recommended, then, that economic policies are oriented towards raising educational attainment.

Keywords: Economic growth, human capital, inequality, Latin-America; JEL: I24, $\mathrm{I} 25, \mathrm{I} 28$

\section{Capital humano, desigualdade e crescimento econômico na América Latina}

Resumo Macarena Suanes e Oriol Roca estudaram os principais determinantes da desigualdade e do crescimento econômico na América Latina entre 1980 e 2009, e descobriram que um dos principais determinantes era o investimento estrangeiro direto. Este artigo prolonga esse estudo até 2015 e mostra que tal investimento já não é significativo. Em contraste, o aumento do capital humano reduz significativamente a desigualdade e melhora o crescimento econômico. Recomenda-se, portanto, que as políticas econômicas sejam orientadas para o aumento de do nível de educação.

Palavras-chave: crescimento econômico, capital humano, desigualdade, América Latina; JEL: I24, I25, I28 
$\mathrm{E}$ ste artículo explora los principales determinantes de la desigualdad y el crecimiento en América Latina. Existe una extensa literatura que identifica diversos mecanismos teóricos mediante los cuales incrementos de la inversión extranjera directa (IED) puede promover el crecimiento económico (ver, p. ej., De Mello, 1997, 1999; y Suanes y Roca, 2015). Aunque hay menos estudios sobre el tema, algunos autores encuentran que la IED es un determinante de la desigualdad. En concreto, Suanes y Roca (2015) encuentran que cuando la IED aumenta, la desigualdad se incrementa hasta un umbral en el que comienza a disminuir para un panel de 18 países de América Latina en el período 1980-2009.

Pero hay otros factores, como el capital humano y la educación, que pueden ser determinantes en la evolución de la desigualdad y del crecimiento. De hecho, la literatura muestra que el capital humano y la educación son potencialmente importantes en la determinación del crecimiento a largo plazo $^{1}$, y en la reducción de la desigualdad (ver Castelló y Doménech, 2002).

En este trabajo, que extiende el estudio de Suanes y Roca (2015) desde 2009 hasta 2015, encontramos que la IED ya no tiene un papel significativo, mientras de los niveles de escolaridad tienen un efecto negativo y positivo significativo en la desigualdad y el crecimiento económico. Además, que las estimaciones puntuales del capital humano arrojan los mayores valores, de modo que el aumento del capital humano es de la mayor importancia para reducir la desigualdad y amentar el crecimiento económico en América Latina, cuya tasa de crecimiento redujo significativamente en 2008 debido a la recesión internacional.

El artículo se divide en cuatro secciones. En la primera se revisan las teorías sobre la relación entre capital humano, inversión extranjera directa, desigualdad y crecimiento. En la segunda se muestra la evolución de esas variables en América Latina. En la tercera se describen los resultados del análisis econométrico y en la última se presentan las conclusiones.

\section{CAPITAL HUMANO, IED, DESIGUALDAD Y CRECIMIENTO}

\section{EL CAPITAL HUMANO Y SU IMPACTO EN EL CRECIMIENTO}

El capital humano cumple un papel clave en diversas teorías del crecimiento. Becker (1974) considera que es el conjunto de talentos

${ }^{1}$ Ver, p. ej. Lucas (1988), Barro (1991), Mankiw et al. (1992); Benhabib y Spiegel (1994), Fernández y Rogerson (1995) y Bénabou (1996a). 
y competencias de los trabajadores productivos, bien sea que los adquieran de manera informal (por la experiencia) o formal (en la educación o formación). También se puede considerar como una inversión, pues aumenta la productividad de las personas.

Una de los propósitos de la economía es entender por qué el crecimiento es desigual entre países, un tema de gran importancia desde la publicación de La riqueza de las naciones, de Adam Smith, en 1776, donde argumentó que la inversión en capital aumenta la productividad futura y que la educación es un determinante de los ingresos y de la productividad individuales. Años después, Mincer (1958), Schultz (1961) y Becker (1964) subrayaron la importancia del capital humano y establecieron conceptualmente esa tesis, argumentando que la educación es un elemento optimizador durante el ciclo de vida.

A finales de los noventa e inicios de este siglo, Caselli et al. (1996) y Pritchett (2001) cuestionaron esa tesis. No encontraron una relación positiva, y en algunos casos encontraron una relación negativa entre educación y crecimiento. Concluyeron entonces que se debía considerar la calidad del sistema educativo. Y cuando lo incluyeron en las regresiones, volvieron a encontrar la relación positiva entre capital humano y crecimiento.

Lucas (1988) y Mankiw et al. (1992) abrieron un nuevo debate sobre la relación entre capital humano y crecimiento. De hecho, Lucas introdujo la actividad educativa como factor de crecimiento a largo plazo y explicó las diferencias de las tasas de crecimiento entre países. Y Mankiw et al. (1992) hicieron una prueba empírica de la hipótesis neoclásica de Solow, en la que evaluaron la eficacia del modelo para explicar las variaciones del nivel de vida. Concluyeron que los datos demográficos y la inversión en capital humano explicaban un aumento del 80\% del PIB en varios países.

Los modelos neoclásicos de crecimiento endógeno destacan la importancia del capital humano en el desarrollo de la economía. Estos modelos se pueden agrupar en dos categorías según su enfoque del vínculo entre capital humano y crecimiento. La primera considera el capital humano como factor de acumulación, así como el capital físico en la función de producción; su acumulación favorece el crecimiento, de modo los niveles de producción difieren entre países debido a sus diferentes niveles de capital humano (Romer, 1990). La segunda considera que un mayor capital humano incide en el crecimiento facilitando la innovación y la adopción de nuevas tecnologías, de modo que las diferencias de capital humano producen diferencias en el crecimiento de la producción (Nelson y Phelps, 1966). 
Barro (1991) estimó que una variación del 50\% al 100\% en la escolaridad secundaria aumentaba la tasa de crecimiento anual de las ganancias en cerca de un punto porcentual. Pero a mediados de los noventa se desvaneció el optimismo sobre la relación entre capital humano y crecimiento. Benhabib y Spiegel (1994) estudiaron el efecto del capital humano o del nivel de educación en el crecimiento y la producción. No encontraron la relación positiva descrita por Mankiw et al. utilizando un enfoque estándar, el de tratar el capital humano, medido por la escolaridad promedio de los trabajadores, como un factor de producción ordinario. Propusieron entonces un enfoque alternativo asociado a la teoría del crecimiento endógeno, que modela el progreso tecnológico la productividad total de los factores (PTF), como función del nivel educativo o del capital humano. La intuición es que los trabajadores más educados son más aptos para crear, aplicar y adoptar nuevas tecnologías, que generan crecimiento. Sus resultados cuestionan el papel tradicional del capital humano como factor separado, que contribuye al crecimiento. Concluye que los países más pobres podrían aumentar su acervo de capital humano y superar a los más ricos. Una de las implicaciones es que el acervo de capital humano influye en la tasa de crecimiento del PIB per cápita más que su tasa de crecimiento. Benhabib y Spiegel también muestran que en los países más ricos, el efecto directo de la educación en la capacidad de innovación es mayor que en los países más pobres; de modo que el impacto de la educación en el crecimiento varía en función del nivel de desarrollo del país.

Otros estudios empíricos, como los de Pritchett (2001) y Krueger y Lindahl (2001), también pusieron en tela de juicio el optimismo sobre la contribución de la educación al crecimiento. Krueger y Lindahl encontraron que solo es positiva y estadísticamente significativa en países con bajo nivel de educación. Pritchett no encontró una relación entre aumento del capital humano y tasa de crecimiento del PIB per cápita en una muestra de los países en desarrollo. Da tres razones posibles: 1) el entorno gubernamental e institucional fue desfavorable para la acumulación de capital y debilitó el crecimiento económico; 2) pocos años de escolarización y baja calidad no aumentan el capital humano; 3) los rendimientos de la educación disminuyeron rápidamente, la oferta de educación aumentó y la demanda se estancó.

Ante los estudios empíricos que no pudieron establecer la relación positiva entre capital humano y crecimiento, otros autores buscaron mejores explicaciones para los inquietantes resultados de finales de los noventa. En su revisión de la literatura, Dessus (2001) da una 
explicación plausible: la calidad del sistema educativo evoluciona de manera diferente entre países y la acumulación de capital humano a un mismo ritmo puede producir resultados muy diferentes. Dessus propone un marco alternativo en el que se supone que la heterogeneidad de la función de producción es captada por la productividad marginal del capital humano y que dotación inicial de capital humano explica las diferencias de calidad del sistema educativo. Así confirma los resultados de Azariadis y Drazen (1990), quienes encontraron que no hay un rápido crecimiento sin un alto nivel de inversión en capital humano con respecto al ingreso per cápita.

Barro (2001) integró la calidad educativa en el modelo de crecimiento endógeno y encontró un papel positivo de la educación en el crecimiento. Créel y Poilon (2006) estimaron el impacto del capital humano (medido por el gasto educativo) y la inversión pública en el crecimiento con un modelo de Solow, y encontraron resultados positivos en Europa. Pero Suanes y Roca (2015) no encontraron ninguna relación significativa entre capital humano y crecimiento en los países latinoamericanos entre 1980 y 2005.

Por su parte, Gunther (2007) encontró que la educación afecta a la vez el crecimiento y la desigualdad de ingresos, que una mayor educación no necesariamente reduce la desigualdad y que no hay una relación clara entre crecimiento y desigualdad. En cambio, Suanes y Roca encuentran una relación negativa y significativa entre capital humano y desigualdad en América Latina.

\section{IMPACTO DE LA IED EN EL CRECIMIENTO Y LA DESIGUALDAD}

Según diversos autores, en las economías en desarrollo, la IED tiene un impacto positivo en el crecimiento económico ${ }^{2}$, pero no es claro su impacto en la desigualdad del ingreso ni si persiste y es alto en América Latina. Otros autores no encuentran una relación entre dichas variables o encuentran una relación negativa ${ }^{3}$.

Suanes y Roca (2015) argumentan que la IED afecta la desigualdad del ingreso, en especial por su impacto en los salarios, y que esta mejoraría si la población se especializa en actividades intensivas en mano de obra no calificada y disminuye la brecha salarial con los trabajadores calificados. Este argumento supone que los países en

${ }^{2}$ Entre ellos, De Mello, (1997, 1999); Borensztein et al. (1998); Li y Liu, (2005); De Vita y Kyaw, (2009); Feeny et al., (2014), Tsai, (1995); Te Velde, (2003); Choi, (2006); Basu y Guariglia, (2007); Herzer et al., (2012); Lin et al., (2013).

${ }^{3}$ Como Adam (2008), Milanovic (2003) y Sylwester (2005). 
desarrollo tienen abundante mano de obra poco calificada (Robertson, 2000). Jensen y Rosas (2007) indican que la IED afecta la desigualdad suministrando capital, lo que reduce su rendimiento total y aumenta los ingresos laborales. Así, a medida que el capital extranjero y el capital nacional compiten por trabajadores domésticos, los salarios aumentan y la rentabilidad de las empresas locales disminuye, y la desigualdad del ingreso disminuye reduciendo la brecha entre los salarios y los ingresos del capital. No obstante, los receptores de IED tienden a pagar una prima salarial a los trabajadores calificados, de modo que puede aumentar la brecha de ingresos entre trabajadores calificados y no calificados y, por tanto, la desigualdad.

En las economías en desarrollo, las empresas extranjeras tienden a localizarse en sectores intensivos en mano de obra calificada, lo que mejora su posición con respecto a los no calificados (Feenstra y Hanson, 1997). También puede aumentar la oferta de trabajadores calificados mediante capacitación y contribuciones específicas a la educación general (transferencia de conocimientos). Según Berman et al. (1998), la IED induce un crecimiento más rápido de la productividad laboral en empresas extranjeras (transferencia de tecnología) y empresas nacionales (efectos secundarios), y si el aumento de productividad es mayor en sectores cualificados aumentará la brecha con otros sectores. Otros autores encuentran que la IED aumenta la desigualdad de salarios y, por ende, la desigualdad del ingresos en Indonesia (Lipsey y Sjöholm, 2004), China (Zhang y Zhang, 2003), Corea (Mah,2002) y México (Aitken et al., 1996; Feenstra y Hanson, 1997).

Mencionemos ahora algunos estudios que usan métodos diferentes, Tsai (1995), en un análisis transversal de 53 economías, estima una relación positiva y significativa entre la IED y la desigualdad. Choi (2006) encuentra que un aumento de la IED aumenta la desigualdad, para un panel de 119 países en el periodo 1993-2002. Para otro panel de 119 economías en desarrollo, Basu y Guariglia (2007) encuentran una relación positiva entre la IED, el crecimiento económico, la desigualdad del ingreso y el capital humano en el periodo 1970-1999. Herzer et al. (2012) usan técnicas de cointegración para analizar el impacto de la IED en la desigualdad del ingreso a largo plazo en Bolivia, Chile, Colombia, México y Uruguay); sus resultados indican la IED tiene un efecto significativo en casi todos estos países. Y un análisis más reciente, de Lin et al. (2013), de una muestra de economías desarrolladas y en desarrollo encuentra un umbral de capital humano por debajo del cual la IED favorece la igualdad de 
ingresos y, en caso contrario, aumenta la desigualdad. En contraste, Milanovic (2003), con datos de encuestas de hogares de 129 países en 1988-1993-1998, encuentra que la IED no afecta la distribución del ingreso. Y Sylwester (2005), con un panel de 29 economías en desarrollo entre 1970 y 1990, no encuentra una relación positiva.

\section{CAPITAL HUMANO, DESIGUALDAD Y CRECIMIENTO EN AMÉRICA LATINA}

\section{EVOLUCIÓN DEL CAPITAL HUMANO}

Según la OCDE, el capital humano es la mezcla de aptitudes y habilidades innatas con la calificación y el aprendizaje que se adquieren en la educación y la capacitación, que reportan beneficios económicos y económicos a largo plazo. El Reporte de Capital Humano 2015 (WEF, 2015) dice que América Latina tiene un problema de "formación y capacitación" de capital humano, que se refleja en su baja productividad y competitividad, y explica por qué no ha tenido un crecimiento más dinámico en las últimas décadas, lo que ha impedido que sus ingresos converjan a los niveles de los países más desarrollados.

Entre los factores que influyen en la productividad y la competitividad, el reporte destaca el progreso tecnológico, que crea nuevos bienes y procesos productivos, y las mejoras en la gestión y administración de las empresas, que les dan mayor capacidad para adaptarse a los mercados y enfrentar los riesgos. Latinoamérica y el Caribe aparecen en la zona media-baja del índice, con un puntaje medio global de 59,9. La brecha entre los países de mejor y peor posición es menor que en las demás regiones. Los de mejor posición son Chile, Uruguay y Argentina. Las dos mayores economías, México y Brasil, aparecen en las zonas media y baja, junto con Perú y Colombia. Los de peor posición son Venezuela y países centroamericanos como Honduras (cuadro 1).

Cuadro 1

Índice de capital humano en América Latina, 2015

\begin{tabular}{llll}
\hline Chile & 71,8 & El Salvador & 66,9 \\
Uruguay & 71,2 & Bolivia & 66,5 \\
Argentina & 71,0 & Paraguay & 65,7 \\
Panamá & 71,0 & Brasil & 64,6 \\
Ecuador & 70,8 & República Dominicana & 62,7 \\
Costa Rica & 69,8 & Guatemala & 61,3 \\
México & 68,5 & Nicaragua & 60,7 \\
Perú & 68,1 & Venezuela & 60,5 \\
Colombia & 67,6 & Honduras & 58,9 \\
\hline
\end{tabular}

Fuente: WEF (2015). 
Gráfica 1

Índice de capital humano por países, 1990-2009 y 2010-2015

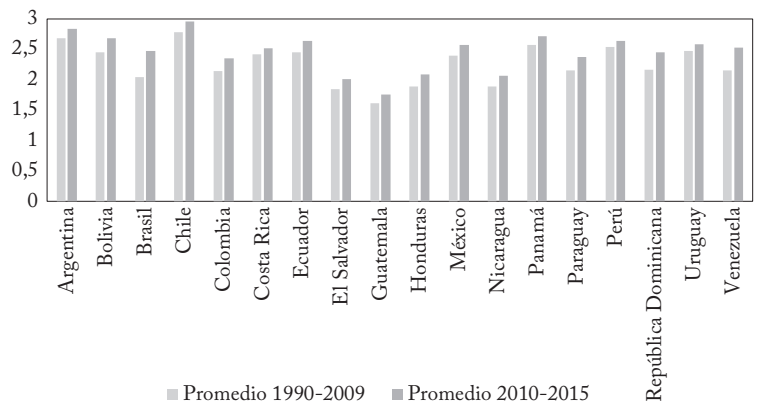

Fuente: Penn World Table 0.0, elaboración propia.

Aunque el índice de capital humano ( $\mathrm{ICH}$ ) revela varias tendencias muestra que los desafíos actuales son educación, habilidades, empleo y perspectivas de futuro, y que los países deben invertir en capital humano para lograr la prosperidad social y económica. La gráfica 1 muestra el promedio del índice considerando la educación secundaria. En el periodo 2010-2015 el índice aumento en todos los países con respecto al periodo 1990-2009.

La gráfica 2 muestra la evolución de la IED en América Latina. Chile, Nicaragua y Panamá muestran los mayores aumentos y Venezuela, la mayor disminución en el periodo de análisis.

\section{Gráfica 2}

IED por países, 1990-2009 y 2010-2015

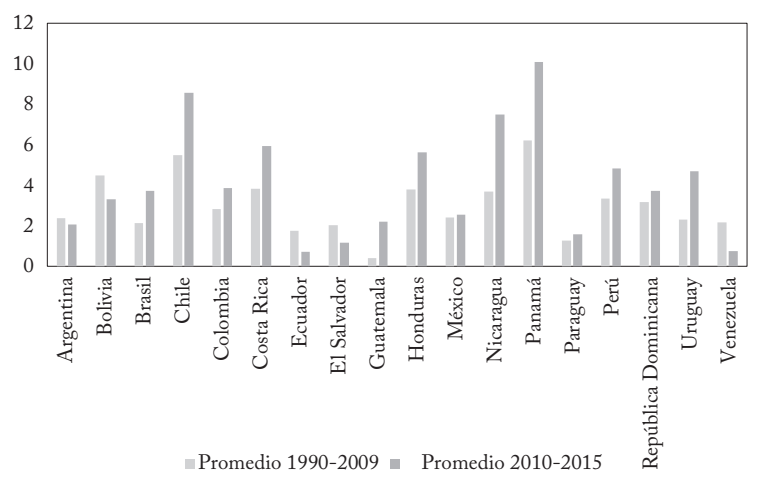

Fuente: World Development Indicators, elaboración propia.

Según la Unctad (2011), América Latina es una de las regiones con mayor IED, un hecho de especial relevancia en una región caracte- 
rizada por crisis políticas y económicas frecuentes, acompañadas de crecimiento acelerado, que han ampliado la brecha con los países desarrollados (Cepal, 2008). La incapacidad para mantener altas tasas de crecimiento durante largos periodos ha impedido resolver los problemas distributivos, una necesidad apremiante para reducir la pobreza (Banco Mundial, 2006).

\section{EVOLUCIÓN DE LA DESIGUALDAD}

En los últimos 15 años ha habido un cambio significativo en los indicadores de desigualdad. En la mayoría de los países empiezan a descender sus niveles con alteraciones graduales, apenas perceptibles en las variaciones interanuales pero evidentes cuando se comparan periodos más largos. Si consideramos el periodo de 1990 a 2015 en los 18 países incluidos en este estudio, vemos mejoras en la distribución, reflejadas en la reducción del coeficiente de Gini en la mayoría de los países (ver gráfica 3).

Gráfica 3

Índice de Gini por países, 1990-2015

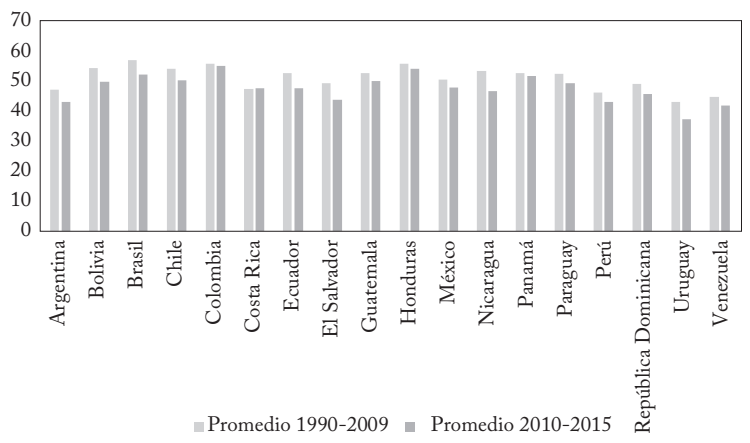

Fuente: UNU-WIDER.

Este cambio de ha sido objeto de diversas interpretaciones. Los ingresos laborales, la principal fuente de recursos de los hogares, han sido determinantes en la reducción de la desigualdad. Más allá de los efectos positivas del aumento del empleo, de la menor tasa de dependencia y de transferencias monetarias redistributivas, el factor que explica la mayor parte de la reducción de la desigualdad es la disminución del diferencial de salarios entre trabajadores cualificados y no calificados (Cepal, 2012). 
Esos cambios distributivos han ocurrido en un contexto político diferente al de las décadas anteriores. La vida democrática ha suscitado nuevas preferencias electorales y dado mayor visibilidad a las demandas sociales. Y los gobiernos han respondido a las demandas de inclusión social con políticas más redistributivas, en un periodo de relativa estabilidad económica (Roberts, 2014).

Otros autores hablan de reformas inspiradas en una "redistribución prudente con crecimiento"(Cornia, 2010), mediante políticas fiscales, laborales y de transferencias progresivas. Las políticas redistributivas y las reformas sociales no han sido exclusivas de los gobiernos izquierdistas, pero la institucionalización de la competencia electoral en contextos de alta desigualdad económica y social parece haber llevado a que partidos y gobiernos de diferentes ideologías respondan a las demandas populares de igualdad e inclusión social (Roberts, 2014).

Así, la característica distintiva de la última década en América Latina ha sido quizá la mejora distributiva. La gráfica 4 muestra la relación entre la inversión extranjera directa y la desigualdad del ingreso, tomando el promedio de ambas variables en el periodo 2010-2015. Y la gráfica 5 muestra la relación entre el índice de capital humano y la desigualdad: cuanto mayor es el índice de capital humano menor es el índice de Gini y, por tanto, menor la desigualdad.

Gráfica 4

IED y desigualdad, 2010-2015

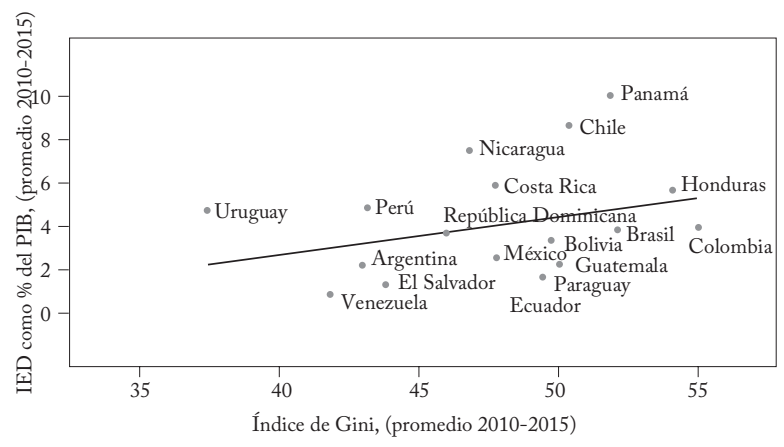

Fuente: World Development Indicators. 


\section{Gráfica 5}

Capital humano y desigualdad, 2010-2015

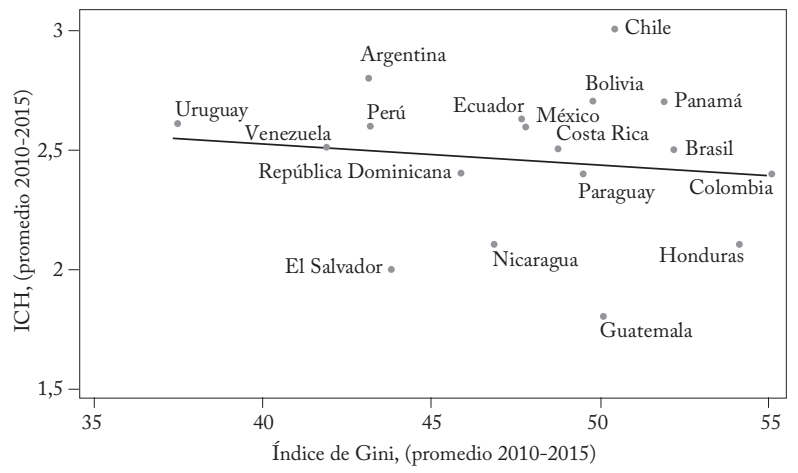

Fuente: World Development Indicators.

\section{EVOLUCIÓN DEL CRECIMIENTO ECONÓMICO}

La gráfica 6 muestra la evolución del crecimiento económico, tomando los promedios de 1990-2009 y de 2010-2015. La gráfica 7 muestra la relación entre IED y crecimiento para ilustrar el cambio de las variables de interés en el periodo analizado, en la cual se representa la tasa promedio de la IED en cada país entre 2010 y 2015, que ha aumentado en forma significativa en todos los países considerados. La gráfica 8 muestra la relación positiva entre el índice de capital humano y el crecimiento en los países de América Latina.

\section{Gráfica 6}

Crecimiento en América Latina, 1990-2009 y 2010-2015

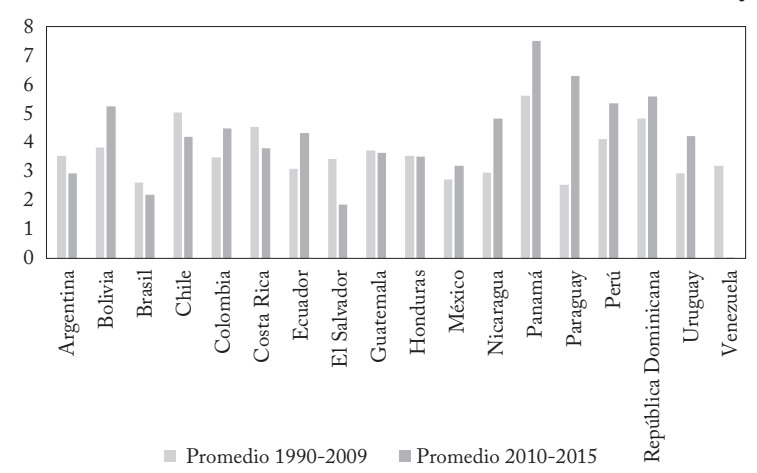

Fuente: World Development Indicators. 
Gráfica 7

Crecimiento e IED, 2010-2015

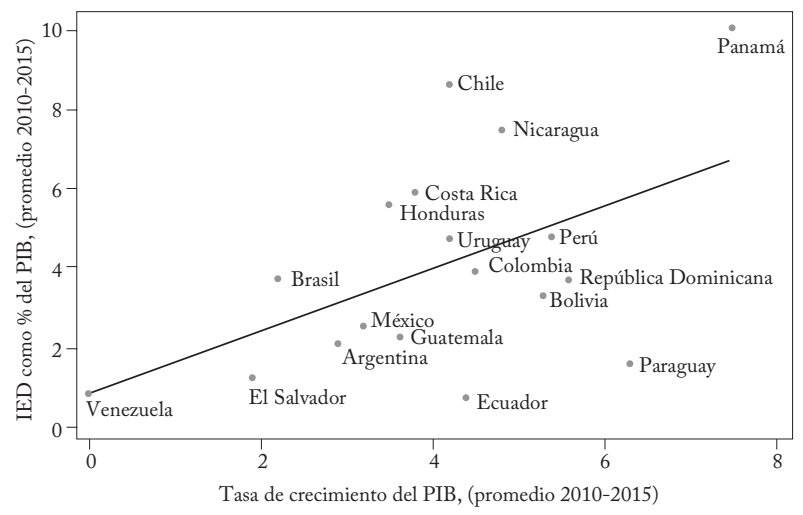

Fuente: World Development Indicators.

Gráfica 8

Capital humano y crecimiento, 2010-2015

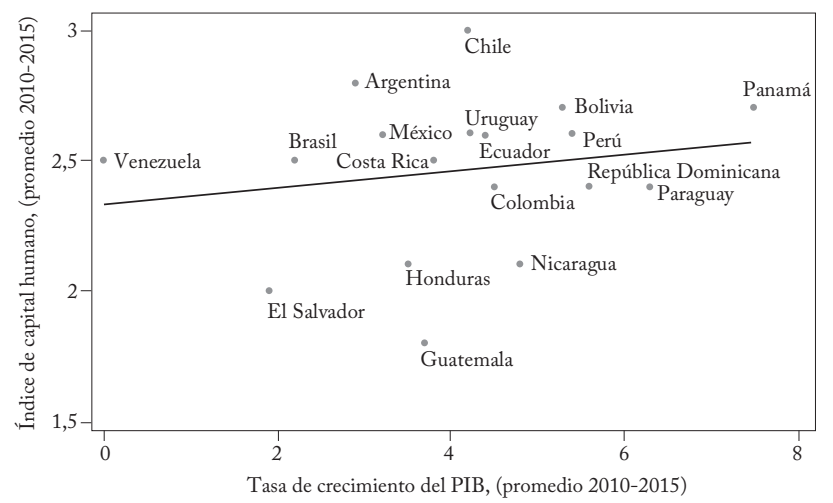

Fuente: World Development Indicators.

\section{DATOS Y ANÁLISIS EMPÍRICO}

Igual que Suanes et al., examinamos los determinantes de la desigualdad y el crecimiento en los mismos países, pero con un panel desequilibrado del periodo 1990-2015 y promedios de tres años. Siguiendo a Figini y Görg (2011), Suanes y Roca (2015) estimamos el modelo:

Desigualdad $_{i t}=\alpha_{0}+\alpha_{1} I E D_{i t}+\alpha_{2} I E D^{2}{ }_{i t}+\alpha_{3} X_{i t}+u_{i}+e_{i t}$

y siguiendo a Basu y Guarriglia (2007), De Mello (1999), Clark et al (2011) y Suanes y Roca (2015), modelamos el crecimiento así: 
Crecimiento $_{i t}=\beta_{0}+\beta_{1} L P I B_{i t-1}+\beta_{2} I E D_{i t}+\beta_{3} X_{i t}+u_{i}+v_{t}+e_{i t}$.

Donde $X_{i t}$ incluye las siguientes variables de control: comercio, población, inversión, gasto público, ICH y oferta monetaria; $u_{i}$ son los efectos fijos de los 18 países; $v_{t}$ son los efectos fijos de los años 1990 a 2015; $e_{i t}$ es el término de error y $L P I B_{i t-1}$ es el PIB inicial. El cuadro 2 muestra las fuentes de los datos.

Cuadro 2

Variables y fuentes

\begin{tabular}{lll}
\hline Variables & Definición de las variables & Fuente \\
\hline Desigualdad & Índice de Gini & UNU-WIDER 2.c \\
Crec. PIB & Crecimiento real del PIB real per cápita & WDI \\
lpib & PIB real per cápita en logaritmos & Cepal \\
IED & Inversión extranjera directa (\% del PIB) & WDI \\
Capital humano & $\begin{array}{l}\text { Porcentaje de la población con educa- } \\
\text { ción secundaria 25 o más del total }\end{array}$ & Penn World Table \\
& Gasto del gobierno (\%del PIB) & WDI \\
Gasto público & Tasa de crecimiento de la población & WDI \\
Población & Tasa de M2 (\% del PIB) & WDI \\
M2 & Inversión bruta en capital fijo (\% del & WDI \\
Inversión & PIB) & \\
Comercio & Importaciones + exportaciones (\% del & Banco Mundial \\
& PIB) & \\
\hline
\end{tabular}

WDI $=$ World Development Indicators.

Las ecuaciones (1) y (2) incluyen una variable ficticia que refleja la recesión internacional que afectó a toda la región en 2008 (Ortiz et al., 2013). E1 cuadro 3 muestra que solo es significativa como determinante del crecimiento económico en los países seleccionados (no se encontró una relación significativa con la evolución del índice de Gini). En ambas ecuaciones probamos si el ICH al cuadrado es relevante para explicar la desigualdad y el crecimiento, pero en ningún caso encontramos una relación estadísticamente significativa.

Igual que Suanes et al., para hacer las estimaciones usamos tres métodos: 1) un modelo con efectos fijos (EF) de país para controlar la heterogeneidad no observable y la posible omisión de variables relevantes. No usamos un modelo de efectos aleatorios porque implicaría que nuestra serie de datos es una muestra aleatoria tomada de una población grande (Suanes et al. 2015).2) estimamos el modelo con el método generalizado de momentos (MGM) propuesto por Arellano y Bond (1991, 1995), que en primeras diferencias permite tener en cuenta la heterogeneidad no observable por países y controlar 
la posible existencia de problemas de endogeneidad, utilizando las variables rezagadas uno o más periodos como variables instrumentales. 3) además usamos mínimos cuadrados en dos etapas (MC2E) para controlar la posible existencia de endogeneidad entre la variable dependiente y la variable explicativa. Como variable instrumental, se tomó el valor de las variables al comienzo del periodo de tres años, que se espera no estén correlacionado con los errores.

Para probar si las condiciones de los momentos son válidas cuando se usa el método MGM, utilizamos el contraste de Arellano-Bond (contraste M2) para la autocorrelación de segundo orden en los errores de primera diferencia (Arellano y Bond, 1991) y Blundell y Bond (1998)). El cuadro 3 muestra los resultados: no se puede rechazar la hipótesis nula de autocorrelación cero en los primeros errores de diferencia en el orden 2, lo que demuestra que las condiciones de momento son válidas.

Los paneles A y B muestran que, en general, la IED no fue estadísticamente significativa para explicar la evolución de la desigualdad en el periodo. Y el Panel B muestra que el cuadrado de la IED nunca fue estadísticamente significativo. Pero, con los tres métodos, el ICH siempre resultó significativo con un signo negativo. También encontramos que las estimaciones puntuales asociadas al capital humano arrojan el mayor valor, de modo que el aumento del capital humano (medido por los años de escuela secundaria) es el principal determinante de la reducción de la desigualdad. Por tanto, en América Latina se debe aumentar el nivel de educación.

Por su parte, el Panel $\mathrm{C}$ indica de nuevo que la IED no es en general estadísticamente significativa para explicar la evolución del crecimiento, mientras que el ICH, independientemente del método de estimación, siempre tiene un efecto grande y positivo. Además, la variable que refleja la recesión internacional de 2008 siempre tiene un efecto negativo y significativo en el crecimiento económico, como también encontraron Ortiz et al. (2013).

\section{CONCLUSIONES}

En este artículo extendemos el estudio de Suanes et al. hasta 2015 para los mismos países, y encontramos que la IED ya no tiene un papel significativo, mientras que la escolarización tiene efectos negativos y positivos significativos en los niveles de desigualdad y crecimiento económico, respectivamente. El aumento del capital humano es el factor que más reduce desigualdad y más aumenta el crecimiento económico, 
y por ello se recomienda que la política pública en América Latina se dirija a aumentar el nivel de educación. Además, como era de esperar, los resultados indican que la recesión internacional de 2008 redujo significativamente el crecimiento económico en América Latina.

Cuadro 3

Resultados de las estimaciones de las ecuaciones

\begin{tabular}{|c|c|c|c|c|c|c|c|c|c|}
\hline \multirow{2}{*}{ Variable } & \multicolumn{3}{|c|}{ Panel A } & \multicolumn{3}{|c|}{ Panel B } & \multicolumn{3}{|c|}{ Panel C } \\
\hline & $\mathrm{EF}$ & $\mathrm{MC} 2 \mathrm{E}$ & MGM & $\mathrm{EF}$ & $\mathrm{MC} 2 \mathrm{E}$ & MGM & $\mathrm{EF}$ & MC2E & MGM \\
\hline \multirow{2}{*}{ IED } & 0,139 & $0,350^{* *}$ & $-0,186$ & $-0,048$ & 0,344 & $-0,467^{* * *}$ & 0,038 & $0,183^{* * *}$ & 0,069 \\
\hline & $(0,139)$ & $(0,162)$ & $(0,180)$ & $(0,301)$ & $(0,350)$ & $(0,226)$ & $(0,114)$ & $(0,091)$ & $(0,170)$ \\
\hline \multirow{2}{*}{ IED2 } & - & - & - & 0,202 & 0,001 & 0,030 & - & - & - \\
\hline & - & - & - & $(0,029)$ & $(0,036)$ & $(0,028)$ & - & - & - \\
\hline \multirow{2}{*}{ LPIBit-1 } & - & - & - & - & - & - & $2,977^{\text {秘水 }}$ & 0,047 & - \\
\hline & - & - & - & - & - & - & $(1,317)$ & $(0,168)$ & - \\
\hline \multirow{2}{*}{ Comercio } & $-0,020$ & $-0,011$ & $-0,004$ & $-0,018$ & $-0,011$ & $-0,009$ & $0,049^{*}$ & 0,016 & $0,086^{*}$ \\
\hline & $(0,021)$ & $(0,013)$ & $(0,024)$ & $(0,022)$ & $(0,013)$ & $(0,026)$ & $(0,017)$ & $(0,010)$ & $(0,027)$ \\
\hline \multirow{2}{*}{ Población } & $-1,358$ & $3,487^{*}$ & $-0,131$ & $-1,480$ & $3,484^{*}$ & $-0,104$ & $2,143^{* * *}$ & 0,387 & 1,328 \\
\hline & $(1,237)$ & $(0,656)$ & $(2,010)$ & $(1,251)$ & $(0,671)$ & $(1,977)$ & $(1,064)$ & $(0,371)$ & $(1,869)$ \\
\hline \multirow{2}{*}{ Inversión } & - & - & - & - & - & - & 0,022 & $-0,007$ & 0,134 \\
\hline & - & - & - & - & - & - & $(0,055)$ & $(0,032)$ & $(0,085)$ \\
\hline \multirow{2}{*}{ Gasto público } & $0,166^{* * *}$ & $0,377^{*}$ & $-0,428^{*}$ & $0,171^{* * * *}$ & $0,377^{*}$ & $-0,451^{*}$ & $-0,252^{*}$ & $-0,156^{*}$ & $-0,502^{*}$ \\
\hline & $(0,097)$ & $(0,097)$ & $(0,141)$ & $(0,097)$ & $(0,099)$ & $(0,153)$ & $(0,078)$ & $(0,055)$ & $(0,119)$ \\
\hline \multirow{2}{*}{$\mathrm{ICH}$} & $-5,947^{\text {*** }}$ & $-4,559^{*}$ & $-12,626^{*}$ & $-5,960^{* *}$ & $-4,559^{*}$ & $-12,604^{*}$ & $3,723^{\text {*ak }}$ & $1,282^{* *}$ & $5,924^{*}$ \\
\hline & $(2,381)$ & $(1,044)$ & $(3,056)$ & $(2,385)$ & $(1,044)$ & $(3,034)$ & $(1,987)$ & $(0,628)$ & $(1,809)$ \\
\hline \multirow{2}{*}{ Oferta monetaria } & $-0,045$ & $0,066^{*}$ & 0,021 & $-0,047$ & $0,066^{* *}$ & 0,028 & $-0,072^{*}$ & $-0,004$ & $-0,021$ \\
\hline & $(0,299)$ & $(0,025)$ & $(0,039)$ & $(0,030)$ & $(0,026)$ & $(0,040)$ & $(0,026)$ & $(0,014)$ & $(0,035)$ \\
\hline \multirow{2}{*}{ Variable ficticia, 2008} & - & - & - & - & - & - & 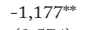 & $-0,831$ & $-1,341^{*}$ \\
\hline & - & - & - & - & - & - & $(0,574)$ & $(0,553)$ & $(0,506)$ \\
\hline \multirow{2}{*}{ Constante } & $66,699^{*}$ & $47,729^{*}$ & $57,726^{*}$ & $67,099^{*}$ & $47,740^{*}$ & $57,948^{*}$ & $-38,436^{*}$ & 0,361 & $-13,542^{\text {***** }}$ \\
\hline & $(6,774)$ & $(2,913)$ & $(11,934)$ & $(6,810)$ & $(2,971)$ & $(12,029)$ & $(14,265)$ & $(2,130)$ & $(7,426)$ \\
\hline Contraste M2 & - & - & 0,333 & - & - & 0,349 & - & - & 0,133 \\
\hline Observaciones & 162 & 162 & 108 & 162 & 162 & 108 & 162 & 162 & 126 \\
\hline R2 ajustado & 0,127 & 0,356 & - & 0,130 & 0,353 & - & 0,220 & 0,187 & - \\
\hline Número de países & 18 & 18 & 18 & 18 & 18 & 18 & 18 & 18 & 18 \\
\hline
\end{tabular}

Variable dependiente: Coeficiente de Gini (Paneles A y B), y PIB per cápita (Panel C); método: efectos fijos (EF), MGM, MC2E; periodo: 1990- 2015.

Países considerados: Argentina, Bolivia, Brasil, Chile, Costa Rica, Colombia, Ecuador, Guatemala, Honduras,

México, Nicaragua, Panamá, Paraguay, Perú, República Dominicana, E1 Salvador, Uruguay y Venezuela.

*: significativo al 10\%. **: significativo al 5\%. ***: significativo al 1\%. Las desviaciones típicas aparecen entre paréntesis y se han calcularon considerando el agrupamiento de la variable país. M2 es el contraste de

Arellano-Bond por autocorrelación de segundo orden en la primera diferencia de los errores. En los paneles A y B se introdujeron el primero y el segundo retardo del índice de Gini en la estimación del MGM, y en el panel C el primer retardo del crecimiento del PIB per cápita.

\section{REFERENCIAS BIBLIOGRÁFICAS}

Adam, S. (2008). Globalization and income inequality: Implications for intellectual property rights. Journal of Policy Modeling, 30(5), 725-735.

Aitken, B., Harrison, A. y Lipsey, R. E. (1996). Wages and foreign ownership: A comparative study of Mexico, Venezuela, and the United States. Journal of International Economics, 4O(3-4), 345-371.

Arellano, M. y Bond, S. (1991). Some tests of specification for panel data: Monte Carlo evidence and an application to employment equations. Review of Economic Studies, 58(2), 277-297.

Arellano, M., Bond, S. y Bover, O. (1995). Another look at the instrumental variables estimation of error component models. Journal of Econometrics, 68, 29-51.

Azariadis, C. y Drazen, A. (1990). Threshold externalities in economic development. The Quarterly Journal of Economics, 105(2), 501-526.

Barro, R. J. (1991). Economic growth in a cross section of countries. Quarterly Journal of Economics, 106(2), 407-444. 
Barro, R. y Lee, J. (2001). Schooling quality in a cross-section of countries. Economica, 68(271), 465-488.

Basu, P. y Guariglia, A. (2007). Foreign direct investment, inequality, and growth. Journal of Macroeconomics, 29(4), 824-839.

Becker, G. (1964). Human Capital: A Theoretical Empirical Analysis with Special Reference to Education. Nueva York: Columbia University Press.

Becker, G. (1974). A theory of social interactions. Journal of Political Economy, 82(6), 1063-1093.

Bénabou, R. (1996). Equity and efficiency in human capital investment. Review of Economics Studies, 63(2), 237-264.

Benhabib, J. y Spiegel, M. (1994). The role of human capital in economic development: evidence from aggregate cross-country data. Journal of Monetary Economics, 34, 143-173.

Berman, E., Bound, J. y Machin, S. (1998). Implications of skill-biased technological change: International evidence. Quarterly Journal of Economics, 113(4), 1245-1279.

Blundell, R. y Bond, S. (1998). Initial conditions and moment restrictions in dynamic panel data models. Journal of Econometrics, 87(1), 115-143.

Borensztein, E., de Gregorio, J. y Lee, J. W. (1998). How does foreign investment affect economic growth? Journal of International Economics, 45(1) 115-135.

Caselli, F., Esquivel, G. y Fernando, L. (1996). Reopening the convergence debate: A new look at cross-country growth empirics. Journal of Economic Growth, 1(3), 363-389.

Castelló, A. y Doménech, R. (2002). Human capital inequality and economic growth: Some new evidence. Economic Journal, 478(112), $187-200$.

Cepal. (2008). Transformación productiva con equidad: 20 años después. Viejos problemas, nuevas oportunidades. Santiago de Chile: Cepal.

Cepal. (2012). Panorama social de América Latina 2010 (LC/G.2514-P). Santiago de Chile: Cepal.

Clark, D. P., Highfill, J. et al. (2011). FDI, technology spillovers, growth and income inequality: A selective survey. Global Economy Journal, 11(2), 1-42.

Cornia, G. A. (2010). Income distribution under Latin America's new left regimes. Journal of Human Development and Capabilities, 11(1), 85-114.

Créel, J. y Poilon, G. (2006). Is public capital productive in Europe? Économie publique, 2(6), 95-115.

Choi, C. (2006). Does foreign direct investment affect domestic income inequality? Applied Economics Letters, 13(12), 811-814.

Dessus, S. (2001). Human capital and growth: The recovered role of education systems. World Bank Policy Research working paper 2632. Washington.

De Mello, L. (1997). Foreign direct investment in developing countries and growth: A selective survey. Journal of Development Studies, 348(1), 1-34.

De Mello, L. (1999). Foreign direct investment-led growth: Evidence from time series and panel data. Oxford Economic Papers, 51(1), 133-151. 
De Vita, G. y Kyaw, K. S. (2009). Growth effects of FDI and portfolio investment flows to developing countries: A disaggregated analysis by income levels. Applied Economics Letters, 16(1-3), 277-283.

Feeny, S., Iamsiraroj, S. y McGillivray, M. (2014). Growth and foreign direct investment in the Pacific Island Countries. Economic Modelling, 37, 332-339.

Feenstra, R. y Hanson, G. H. (1997). Foreign direct investment and relative wages: Evidence from Mexico's maquiladoras. Journal of International Economics, 42(3), 371-393.

Fernández, R. y Rogerson, R. (1995). On the political economy of education subsidies. Review of Economic Studies, 62(2), 249-262.

Figini. P y Görg, H. (2011). Does foreign direct investment affect wage inequality? An empirical investigation. The World Economy, 34(9), 1455-1475.

Gunther, R. (2007). Education, economic growth and measured income inequality. Economica, 74(295), 493-514.

Herzer, D., Hühne, P. y Nunnenkamp, P. (2012). FDI and income inequality: Evidence from Latin American, documento de trabajo 1791. Kiel Institute for the World Economy.

Islam, N. (1995). Growth empirics: a panel data approach. Quarterly Journal of Economics, 110(4), 1127-1170.

Jensen, N. M. y Rosas, G. (2007). Foreign direct investment and income inequality in Mexico, 1990-2000. International Organization, 61(3) 467-487.

Krueger, A. y Lindahl, M. (2001). Education and growth: Why and for whom? Princeton working paper No. 429, Princeton University.

Li, X. y X. Liu. (2005). Foreign direct investment and economic growth: An increasingly endogenous relationship. World Development, 33(3), 393-407.

Lin, S.-C., Kim, D.-H. y Wu, Y.-C. (2013). Foreign direct investment and income inequality: Human capital matters. Journal of Regional Science, 53(5), 874-896.

Lipsey, R. E. y Sjöholm, F. (2004). Foreign direct investment, education and wages in Indonesian manufacturing. Journal of Development Economics, 73(1), 415-422.

Lucas, R. E. (1988). On the mechanics of economic development. Journal of Monetary Economics, 22(1), 3-42.

Mah, J. S. (2002). The impact of globalization on income distribution: The Korean experience, Applied Economics Letters, 9(15), 1007-1009.

Mankiw, N. G., Romer, D. y Weil, D. N. (1992). A contribution to the empirics of economic growth. Quarterly Journal of Economics, 152, 407-437.

Milanovic, B. (2003). Can we discern the effect of globalization on income distribution? Evidence for household surveys. Working paper research 2876. World Bank.

Mincer, J. (1958). Investment in human capital and personal income distribution. Journal of Political Economy, 66(4), 281-302. 
Nelson, R. y Phelps, E. (1966). Investment in humans, technological diffusion and economic growth. American Economic Review, 56(1-2), 69-75.

Ortiz, C., Uribe, J. y Vivas, H. (2013). Productividad, acumulación y deseconomías públicas en el crecimiento económico colombiano. Cuadernos de Economia, 32(59), 233-263.

Pritchett, L. (2001). Where has all the education gone? World Bank Economic Review, 15(3), 367-391.

WEF. (2015). The human capital report 2015. Ginebra: World Economic Forum.

Romer, P. (1990). Endogeneous technical change. Journal of Political Economy, 98(S5), 71-102.

Roberts, K. (2014). The politics of inequality and redistribution in Latin America's post-adjustment era. En G.A. Cornia (ed.), Falling inequality in Latin America. Policy changes and lessons Falling inequality in Latin America. Policy changes and lessons (pp. 49-72). Corydon: Oxford University Press.

Robertson, R. (2000). Trade liberalization and wage inequality: Lessons from the Mexican experience. The World Economy, 23(6), 827-849.

Schultz, T. (1961). Investment in human capital. American Economic Review, 51(1), 1-17.

Smith, A. (1958). Investigación sobre la naturaleza y causas de la riqueza de las naciones [1776]. México DF: Fondo de Cultura Económica.

Suanes, M. y Roca S., O. (2015). Inversión extranjera directa, crecimiento económico y desigualdad en América Latina. El Trimestre Económico, 83(3), 675-706.

Sylwester, K. (2005). Foreign direct investment, growth and income inequality in less developed countries. International Review of Applied Economics, 19(3), 289-300.

Te Velde, W. D. (2003). Foreign direct investment and income inequality in Latin America: Experiences and policy implications. Londres: Overseas Development Institute.

Tsai, P. L. (1995). Foreign direct investment and income inequality: Further evidence, World Development, 23(3), 469-483.

Unctad. (2011). World investment report 2009. Nueva York: ONU.

World Bank. (2006). Reducción de la pobreza y crecimiento: círculos virtuosos y círculos viciosos, Estudios del Banco Mundial sobre América Latina y el Caribe. Washington: Banco Mundial.

Zhang, X. y Zhang, K. H. (2003). How does globalization affect regional inequality within a developing country? Evidence from China. Journal of Development Studies, 39(4), 47-67. 